\title{
Process and Outcome Evaluation of Vocational Rehabilitation Interventions in Patients with Prolonged Fatigue Complaints
}

\author{
Margot Joosen • Monique Frings-Dresen • Judith Sluiter
}

Published online: 25 May 2010

(C) The Author(s) 2010. This article is published with open access at Springerlink.com

\begin{abstract}
Background Prolonged fatigue can cause physical, mental and occupational disability. Fatigue often persists because of a combination of biopsychosocial factors.

Purpose To evaluate the process and outcomes of three existing outpatient vocational rehabilitation interventions (VRI) in patients with prolonged fatigue complaints. The VRIs differ with regard to the content and treatment duration, enrolment procedure and financing.

Method A pre-post design was used with repeated measurements before treatment, after treatment and 3 months after treatment. Primary outcomes (fatigue and work participation) and secondary outcomes (physical and social functioning, mental health and physiological indicators (heart rate variability)) were assessed over time using linear mixed models analysis. A process evaluation (i.e. patient reach, content completeness and patient satisfaction) was conducted as well. Results One hundred patients participated. Post-treatment, fatigue decreased $(p<.001)$ and work participation $(p<.010)$, physical functioning $(p<.001)$ and mental health $(p<.001)$ improved considerably in all three VRIs. Social functioning improved in one VRI $(p=.022)$, but did not in the other two ( $p=.442, p=.196$, respectively). Physiologically, heart rate variability improved in two VRIs $(p=.044, p=.038$, respectively). VRIs were administered according to the programme protocol. Almost all patients met their personal goals and the majority was satisfied with the outcomes of diminished constraints at work.

Conclusion Three VRIs showed significant and clinically relevant outcomes over time regarding decreased fatigue and improved functioning and work participation in fatigued
\end{abstract}

M. Joosen $(\bowtie) \cdot$ M. Frings-Dresen $\cdot$ J. Sluiter Coronel Institute of Occupational Health,

Academic Medical Center, University of Amsterdam, P.O. Box 22700, 1100 DE Amsterdam, the Netherlands

e-mail: m.c.joosen@amc.nl patients. The VRIs administered patient-tailored biopsychosocial interventions as planned and patients were satisfied with the interventions.

Keywords Biopsychosocial intervention · Participation . Process evaluation . Prolonged fatigue complaints .

Return-to-work $\cdot$ Vocational rehabilitation

\section{Introduction}

It is known that fatigue is a common complaint in the general and working population, both in healthy people and those in ill health [1]. In the general population, the prevalence of severe fatigue complaints is $10 \%$ to $20 \%$, while in the Dutch working population it is up to $22 \%[2,3]$. However, the definition and origin of fatigue are elusive, even after years of research [4]. Fatigue can be seen as a discrete disorder (e.g. chronic fatigue syndrome (CFS)) [5], a symptom of chronic diseases [1], or as a uni-dimensional continuum of complaints [4]. In this latter view, fatigue can develop from common mild complaints of tiredness to severe disabling fatigue that is not task-specific or easily reversible $[4,5]$. Prolonged severe fatigue complaints often affect individual, social and occupational functioning and would appear to be of multi-factorial origin $[1,5]$. Hence, using a traditional medical model of disease to explain prolonged fatigue would be insufficient [6]. The biopsychosocial model [7], which states that sickness and health results from a complex interaction between biological, psychological and social factors, seems to be more appropriate to explain prolonged fatigue and is used by several fatigue researchers $[8,9]$.

In line with the biopsychosocial model, mechanisms responsible for the perpetuating character of prolonged fatigue have been investigated. From a (neuro)biological point of view, prolonged exposure to stress, accompanied by the inability to 
terminate stress responses, may lead to overuse and damage of physiological stress systems [10]. Relationships between dysregulation of stress systems (i.e. autonomic nervous system) [11, 12], HPA axis hypofunction [13] and prolonged fatigue complaints have been reported. Secondly, cognitive and behavioural factors are found to be involved in the perpetuation of fatigue complaints $[14,15]$. These perpetuating factors are important for designing an appropriate intervention [16]. Treatment should therefore include physical, psychological or cognitive behavioural and (psycho)social interventions. To be more specific, intervening on those factors that perpetuate symptoms of the individual patient should be the target of interventions [17]. This approach has already shown positive effects in RCT research in CFS [9] and cancer-related fatigue [18] and in treating CFS patients in clinical practice [19]. Furthermore, intervention research in fatigued patients directed towards symptoms and complaints has been done [20, 21]. However, research focussing on participation, especially work participation, is scarce.

In the Netherlands, multicomponent interventions are being offered to workers with participation problems [20, 22]. Although these vocational rehabilitation interventions (VRIs) are practiced, there is a lack of evidence for the effects and content of these treatments. In this study, three different existing VRIs for fatigue patients are evaluated. When studying interventions in their usual settings, researchers are not able to manipulate content and organisation [23]. A process evaluation provides understanding of how an intervention was conducted and received [24].

The aim of this study, therefore, is to assess the outcomes of three VRIs up to 3 months after completion in patients with prolonged fatigue. A second aim is to evaluate the process of the interventions, to gain insight into the outcomes and the content of the interventions. Research questions are as follows:

1. How were the VRIs conducted, in terms of reach of the target population, completeness of the administered intervention and patient's satisfaction?

2. Will fatigue complaints decrease and work ability and work participation increase after completion of the VRIs?

3. Will physical and social functioning, mental health and physiological indicators show positive changes after completion of the VRIs?

\section{Method \\ Participants}

The research population included patients enrolled in one of the three selected VRIs during the period 2006-2008. The VRIs used inclusion criteria to select their clients before treatment, including: good command of spoken and written
Dutch, being motivated to take part in the intervention and not suffering from a psychiatric disorder. From this population, patients between the age of 18 and 60 years who reported to have fatigue complaints (for a longer period of time) as a main or important symptom and suffering from functional impairments (i.e. constraints in everyday life) due to fatigue complaints were eligible for participating in the study. These inclusion criteria were checked by a supervisor of the VRI during an intake procedure. Eligible patients were approached and informed about the study before they provided written consent. This study was approved by the Medical Ethical Committee of the Academic Medical Center.

\section{Design}

Three institutions providing outpatient VRIs participated in this study. All three saw patients with prolonged fatigue complaints and problems with (work)participation. Differences between the institutions included content and duration of the treatment programmes, enrollment procedures and financing of treatment (Table 1).

A pre-post design with repeated measurements was carried out at each of the sites. Measurements took place at baseline (t0), upon completion of the intervention (t1) and 3 months after completing the intervention ( $t 2)$. The results observed during $\mathrm{t} 1$ and $\mathrm{t} 2$ were compared with respect to baseline results. Because it is likely that the three VRIs had different target populations (including factors such as motivation, sick leave duration and disability level), it was not possible to compare the intervention outcomes of the three VRIs. In addition to evaluating outcomes of the VRIs, a process evaluation was conducted to assess the process and content of each intervention.

\section{Vocational Rehabilitation Interventions}

\section{VRI 1}

The first VRI was carried out in a regional outpatient centre (Energy Control), mainly consisting of physical training, relaxation and breathing exercises. Based on an exercise test, in which individual heart rate levels were registered, a progressive personal workout scheme was developed to improve physical/aerobic fitness. For a detailed description of the intake procedure, see Joosen et al. [20]. During the training, heart rate values were monitored and used as references to determine the load of the training. VRI1 took 18 weeks, with a centre visit frequency of three times a week (Table 1).

\section{VRI 2}

A regional vocational outpatient institution (Reaplus) carried out a multicomponent treatment programme. The programme 
Table 1 Characteristics of the three vocational rehabilitation interventions (VRIs)

\begin{tabular}{|c|c|c|c|}
\hline & VRI 1 & VRI 2 & VRI 3 \\
\hline Target group & $\begin{array}{l}\text { Patients with fatigue complaints and } \\
\text { functional impairments }\end{array}$ & $\begin{array}{l}\text { Workers with fatigue complaints and } \\
\text { functional (work) impairments }\end{array}$ & $\begin{array}{l}\text { Workers with fatigue complaints and } \\
\text { functional (work) impairments }\end{array}$ \\
\hline $\begin{array}{l}\text { Recruitment } \\
\text { strategies }\end{array}$ & Self referred & $\begin{array}{l}\text { Referred by an occupational physician } \\
\text { or benefits agency }\end{array}$ & $\begin{array}{l}\text { Referred by an occupational physician } \\
\text { or benefits agency }\end{array}$ \\
\hline $\begin{array}{l}\text { Financing of the } \\
\text { intervention }\end{array}$ & By patients themselves & By the employer of the patient & By the employer of the patient \\
\hline Overall goal & $\begin{array}{l}\text { Reduce fatigue complaints and regain } \\
\text { balance between activity and rest }\end{array}$ & $\begin{array}{l}\text { Achieving a normal balance between } \\
\text { daily life and work }\end{array}$ & $\begin{array}{l}\text { Achieving a normal pattern of } \\
\text { functioning including return-to-work }\end{array}$ \\
\hline Duration & 18 weeks & 18 weeks ( 3 x6 weeks) & 4,5 or 6 weeks \\
\hline Days & 3 times a week & $3,2,1$ times a week & From 5 to 2 times a week \\
\hline Level & Individual & Individual and group level & Group and individual level \\
\hline
\end{tabular}

consisted of physical training (i.e. progressive personal workout scheme was used based on an exercise test in which heart rate levels were registered), psychological and cognitive behavioural sessions and return-to-work sessions [22]. VRI2 took 18 weeks, with a frequency of three times a week during the first 6 weeks, decreasing from two to one time per week for the following 6 weeks. During the third period, patients were expected to engage in physical training two times per week (Table 1).

\section{VRI 3}

The third VRI, a multicomponent treatment programme, was carried out by a national vocational outpatient institution (Winnock). Four of its ten locations were included for this study and followed the same protocol. The duration of the programme depended on the duration of sick leave; the intervention was set at four weeks when the patient was absent from work less than 18 consecutive weeks, 5 weeks at 18-26 weeks sickness absence and 6 weeks when absence duration was longer than 26 weeks. The first 1, 2 or 3 weeks of intervention consisted of a 5 days-a-week programme of cognitive behaviour therapy principles, physical training (i.e. graded activity using time-contingent training) and return-to-work sessions. Frequency diminished to one or two times a week in the weeks following (Table 1).

\section{Process Evaluation}

Based on key elements in process evaluation research, i.e. reach, dose delivered and dose received, an evaluation was conducted on each programme intervention [24]. Reach addressed (1) patient characteristics (i.e. demographics, degree of fatigue, duration of fatigue and disability), collected at baseline by means of a self-administered questionnaire; and (2) data about frequency of and reasons for intervention dropouts were collected by the researcher during the intervention period.
Dose delivered analysis reviewed whether the interventions were administered as planned. Following each session or training day, supervisors or trainers completed a structured process evaluation form. Presence of patients and reasons for not attending meetings and intervention components delivered of the specific session were registered for each participant. Frequency of components delivered was compared with the programme protocol to check the completeness of the interventions. Completeness was determined to be 'full' when more than $74 \%$ of the prescribed amount of components took place, 'fair' between 50-74\% and 'incomplete' when less than $50 \%$ of the programme protocol was delivered.

Dose received, as measured by patient satisfaction, was recorded at $\mathrm{t} 1$. Personal goals were evaluated through openended questions and the perceived effectiveness of the intervention on experienced constraints at work was also assessed by questionnaire.

\section{Outcome Evaluation}

Data to assess the outcomes of the VRIs were collected at $\mathrm{t} 0, \mathrm{t} 1$ and $\mathrm{t} 2$. Outcome variables were divided into primary outcomes and secondary outcomes.

\section{Primary Outcomes}

To investigate fatigue complaints, three questionnaires were used. Firstly, severity of fatigue complaints was measured with the Checklist Individual Strength (CIS) [25], which consists of 20 statements that cover several aspects of fatigue. The total score was calculated by adding all item scores and ranged from 20 to 140. Higher scores indicated a higher severity of fatigue. The CIS has been validated in the Dutch working population [26]. The Vitality subscale, one subscale of the Dutch version of the RAND-36 Health survey [27], which is almost identical to the MOS SF-36 [28], was used to measure fatigue as well. The score ranged from 0 to 100 , higher scores being better. The RAND-36 is a reliable and validated generic instrument [27]. Third, work-related fatigue was measured 
with the Need for Recovery After Work scale [29]. This scale comprises 11 dichotomous items. The total score was calculated by adding the item scores and transformed into a scale ranging from 0 to 100 . Higher scores indicated a higher degree of need for recovery after work. The Need for Recovery scale was found reliable in a working population [30].

Data on work participation were collected at $\mathrm{t} 0, \mathrm{t} 1$ and $\mathrm{t} 2$ by researcher-formulated questionnaire. Data consisted of (1) current work status, in terms of employed or unemployed; (2) number of contractual hours; and (3) absolute number of hours the patient was working. These data were used to determine the percentage of return-to-work, defined as the mean percentage of return to original working hours at $t 0$.

Perceived work ability was assessed using two items from the Work Ability Index (WAI) [31]: (1) current work ability compared with lifetime best, scored between 0 ('not being able to work') and 10 ('lifetime best work ability'); and (2) personal prognosis of work ability in the next two years, scored on a three-point scale ('hardly able to work', 'not sure', 'fairly sure to be able to work').

\section{Secondary Outcomes}

Subscales of the RAND-36 Health survey [27] were used to measure physical functioning and physical role limitation, mental health and emotional role limitation, and social functioning. The scores on each scale ranged from 0 to 100 , higher scores being better.

Heart rate variability (HRV) was used as a physiological indicator and is a marker that reflects sympathetic and parasympathetic activity of the autonomic nervous system. Prolonged exposure to stress, can lead to dysregulation of this system (i.e., lower parasympathetic activity) [10, 32] and can be identified by decreased HRV [33]. HRV was recorded using the Co2ntrol (Decon Medical Systems, Weesp, the Netherlands), a small device attached to a chest strap that detects beat-to-beat intervals of the heart rate signal [20]. HRV was measured at t0 and tl (only in patients of VRI1 and VRI2) during a standardised test protocol: five minutes seated in a resting position for adaptation, followed by $12 \mathrm{~min}$ light exercise on a bicycle ergometer using a single load of $50 \mathrm{~W}$ with a pedal frequency between 60 and $65 \mathrm{~min}^{-1}$. The Co2ntrol was developed according to the guidelines of the European and North American Task Force (1996) [34]. It was found to provide reproducible HRV measurements in healthy individuals and in patients with prolonged fatigue [35].

\section{HRV Data Reduction}

To define HRV, raw data were transferred to HRV Analysis Software version 2.0 (http://venda.uku.fi/research/biosignal), data artefacts were detected and processed by the software.
The data were de-trended using the smoothn prior option. To determine the spectrum of HRV, the Fast Fourier Transform option was used and data were re-sampled at a rate of $4 \mathrm{~Hz}$ using cubic interpolation. The final $9 \mathrm{~min}$ of the 12-min recording period during light exercise were selected. HRV was measured by means of heart period high frequency (HF) power. HF power values were used to estimate respiratory sinus arrhythmia (RSA), the variability of heart period in the respiratory frequency band. RSA is considered a valid index of changes in cardiac vagal tone, which interacts with parasympathetic activity [32, 33]. In this study, HF power was computed in the $0.15-0.5 \mathrm{~Hz}$ respiration window. The VRIs were expected to have beneficial effects on physiological status (i.e. raise HF power after completion of the interventions).

\section{Statistical Analysis}

Statistical analyses were performed using SPSS version 16.0 for Windows (SPSS Inc., Chicago, IL, USA). Values of $p<.05$ were considered statistically significant. To analyse the changes of the three VRIs on the outcome variables over time, linear mixed-model analyses were performed based on repeated measures. The best fitting covariance-variance model was tested before the analysis was applied. A post-hoc procedure (Bonferroni correction: unadjusted $p$ value times 2 ) was followed to test significant differences between test moments ( $\mathrm{t} 1$ and $\mathrm{t} 2$ compared with t0). Analyses were conducted for each VRI separately. To assess the changes in HRV over time, a paired $t$ test was carried out.

\section{Results}

Process Evaluation

Reach

In total, 35 patients enrolled in VRI1, 30 patients in VRI2 and 45 patients enrolled in VRI3. Due to dropouts, analyses were performed for 30, 29 and 41 patients, respectively. See Fig. 1 for a flowchart and reasons for dropout.

Patient's characteristics by VRI group are shown in Table 2 . In all three interventions patients had, on average, severe disabling fatigue complaints for many years (mean over 2.5 years). In the VRI1 and VRI2 groups, most patients (58\% in each group) were on sick leave at baseline. In the VRI3 group, most patients (53\%) were fully at work at baseline.

\section{Dose Delivered}

On average, the VRI1 participants missed three training days because of illness, vacations or bad weather/traffic. In 
Fig. 1 Flow-chart of patients through the study
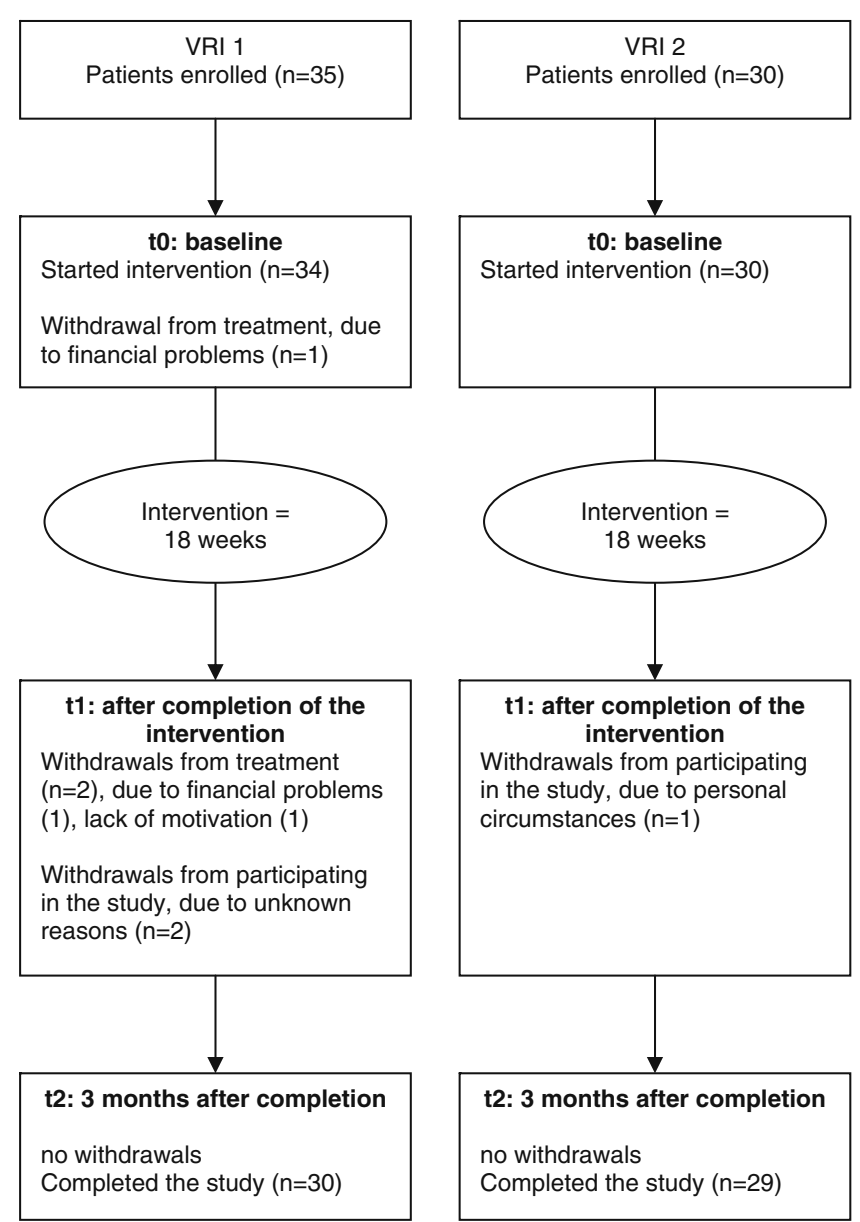

five patients, the programme protocol was adjusted from three times a week to two times a week due to time constraints. In the VRI2 group, patients missed on average four sessions. Reasons for not attending a session were primarily illness and vacation. During the intake procedure, individual goals were set and the programme protocol was tailored to participant needs. The duration of the VRI3 programme depended on the participant's sick leave duration; 21 patients attended the programme for 4 weeks, 10 for 5 weeks and 14 patients attended for 6 weeks.

Table 2 Characteristics of the patients of VRI1, VRI2 and VRI3

\begin{tabular}{llll}
\hline & VRI 1 & VRI 2 & VRI 3 \\
\hline & Mean (SD) or Freq. (\%) & Mean (SD) or Freq. (\%) & Mean (SD) or Freq. (\%) \\
Intervention completed $(n)$ & 30 & 29 & 41 \\
Age & $47(8.1)$ & $45(12.1)$ & $45(10.4)$ \\
Male & $5(17 \%)$ & $16(55 \%)$ & $24(59 \%)$ \\
Female & $25(83 \%)$ & $13(45 \%)$ & $17(41 \%)$ \\
Severe fatigue (CIS score $>76)^{\text {a }}$ & $83 \%$ & $90 \%$ & $95 \%$ \\
Duration of fatigue complaints in years & $5.8(5.6)$ & $3.0(3.6)$ & $3.7(4.7)$ \\
Duration functional impairments in years & $4.0(4.7)$ & $2.5(3.2)$ & $3.4(5.4)$ \\
Employment status & & & $20(49 \%)$ \\
Paid job, fully at work & $10(33 \%)$ & $11(38 \%)$ & $15(37 \%)$ \\
Paid job, partly on sick leave & $8(27 \%)$ & $13(45 \%)$ & $3(7 \%)$ \\
Paid job, fully on sick leave & $6(20 \%)$ & $2(7 \%)$ & $3(7 \%)$ \\
No paid job & $6(20 \%)$ & $3(10 \%)$ & \\
\hline
\end{tabular}

${ }^{a}$ CIS score above 76 was set as the cut-off point for chronic fatigue and increased risk of sickness absence due to fatigue [36] 


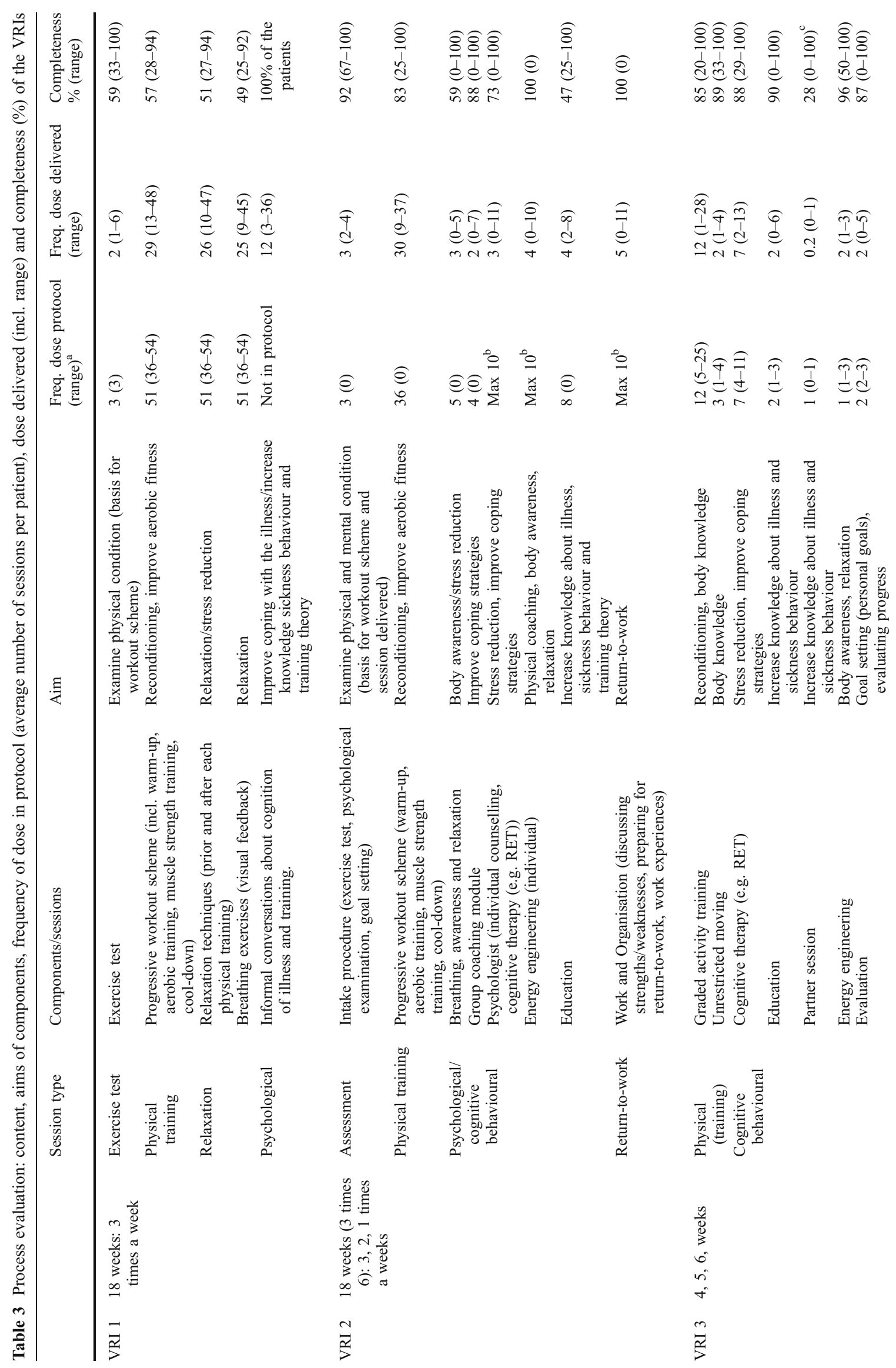


The components of the VRIs, intended frequency per patient, frequency actually delivered and completeness are shown in Table 3. Varieties in the programme protocol (duration and/or components) per patient are taken into account. On average, the completeness of three out of five components of VRI1 was 'fair'. In VRI2 and VRI3, all but one session were carried out as planned ('fair' to 'full' completeness).

\section{Dose Received (Data Not Shown)}

In total, 29 patients in the VRI1 group set personal goals, such as improving energy level or physical condition or being able to relax more. At t1, 24 patients $(83 \%)$ stated their targets were partly to fully met. Out of 28 patients in the VRI2 group, $24(86 \%)$ had partly or fully met their goals at t1; stated goals included improving energy level or physical condition, being able to relax more, improving physical and mental functioning and/or improving work participation. In the VRI3 group, 36 patients set personal targets, including improving energy level, concentration, cognitive functioning, general health and/or work participation. At t1, 34 (94\%) had partly or fully met their goals.

Furthermore, $16(55 \%)$ out of 29 patients in the VRI1 group stated that the intervention was effective in diminishing perceived constraints at work. In the VRI2 sample, this figure was 20 (74\%) out of 27; and in VRI3 participants, $26(68 \%)$ out of 38 patients stated the intervention was effective on constraints at work.

Outcome Evaluation

Primary Outcomes

In Table 4, mean scores with confidence intervals and overall $p$ values for primary outcomes are reported per VRI per measurement.

In all VRIs, fatigue (i.e. fatigue severity, vitality, workrelated fatigue) significantly decreased over time (between $p<.001$ and $p=.001)$. In the VRI1, VRI2 and VRI3 groups, $83 \%, 90 \%$ and $95 \%$, respectively, of patients had CIS scores above the cut-off scores (indicating chronic fatigue) [36] at baseline. At t2, these percentages diminished to $19 \%, 46 \%$ and $50 \%$, respectively.

Significant increases were found in percentage of returnto-work (between $p<.001$ and $p=.01$ ) and in perceived work ability $(p<.001)$ for all VRIs over time. As for the personal prognosis of work ability, in the VRI1 group, 13 $(39 \%)$ out of 33 patients stated at baseline that they were "fairly sure" they would be able to work in two years. At t1, this figures was $21(72 \%)$ out of 29 patients. Out of 30 patients in the VRI2 group, $15(50 \%)$ were 'fairly sure' to 


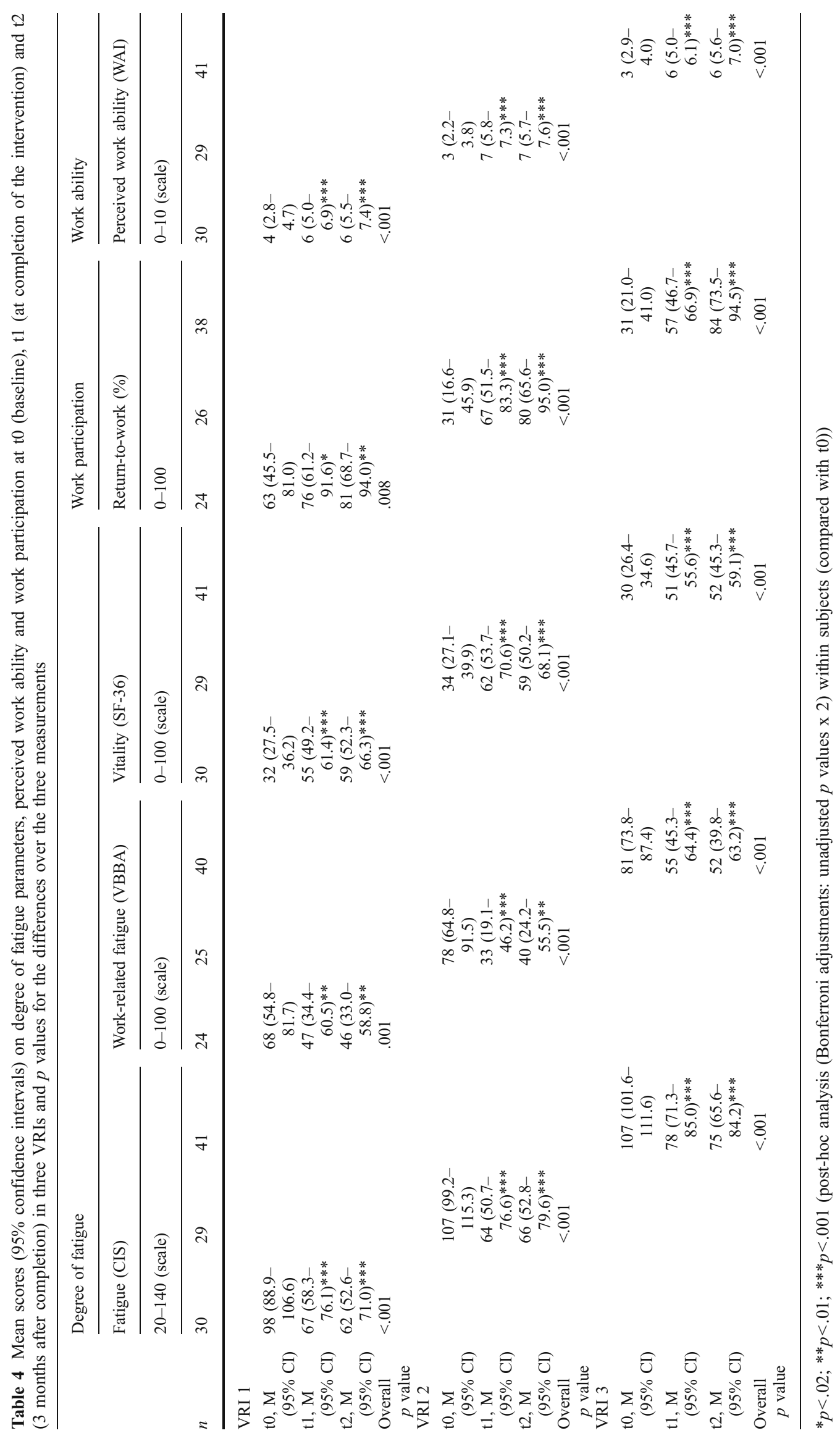




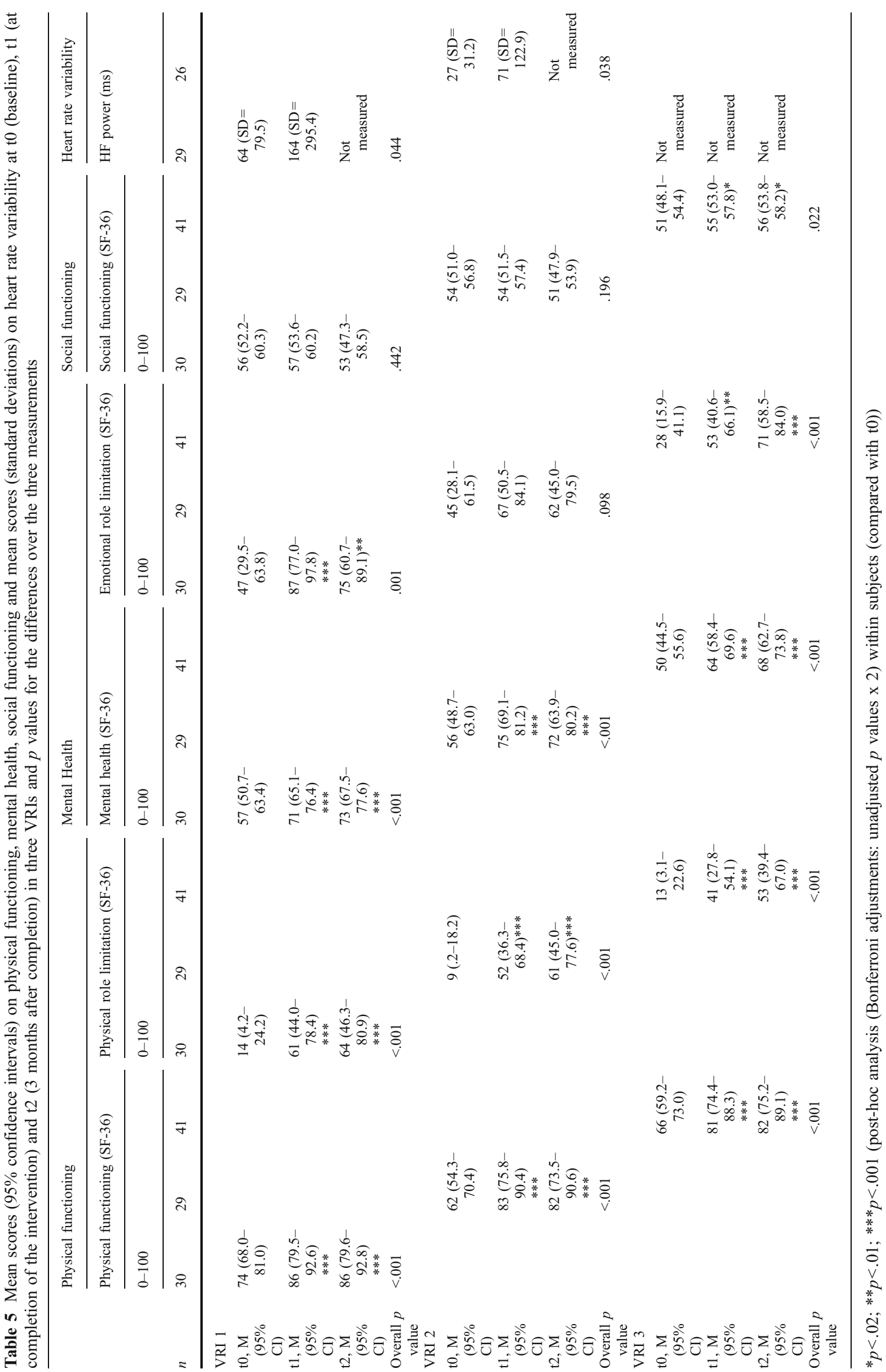


be able to work in two years at baseline, and at $\mathrm{t} 1$, this figure was $21(78 \%)$ out of 27 . While in the VRI3 group, $24(55 \%)$ out of 44 patients at baseline and $26(65 \%)$ out of 40 at $\mathrm{t} 1$ felt this way (data not shown).

\section{Secondary Outcomes}

Table 5 shows mean scores and confidence intervals for secondary outcomes (standard deviations for heart rate variability) and overall $p$ values per measurement for each VRI.

Physical functioning, physical role limitation and mental health improved significantly $(p<.001)$ over time in all three VRI groups. Emotional role limitation improved significantly over time in the VRI1 and VRI3 groups $(p=.001$ and $p<.001$, respectively). Non-significant changes in emotional role limitations were found in VRI2 group participants $(p=.098)$. Social functioning showed non-significant changes in VRI1 and VRI2 participants ( $p=.442$ and $p=.196$, respectively) and significant changes in VRI3 $(p=.022)$. Post-hoc analysis in VRI3 group participants showed significant $(p=.013)$ improvements in social functioning from 51 at baseline to 56 at $\mathrm{t} 2$.

Heart rate variability, i.e. HF power values, increased significantly in the VRI1 and VRI2 groups $(p=.044$ and $p=.038$, respectively) after completion of the interventions.

\section{Discussion}

The process and outcomes of three existing vocational rehabilitation interventions aimed at improving individual and occupational functioning in fatigued patients using a biopsychosocial approach were studied. All interventions were conducted in a sample of patients with prolonged disabling fatigue. The most important components of each intervention were administered as planned, and few dropouts were reported. Furthermore, the majority of the population met their personal goals and stated that the attended intervention was effective in diminishing constraints at work. After treatment and 3 months later, patients had on average significantly fewer fatigue complaints and had improved their individual and occupational functioning. Two secondary outcomes showed no significant effects in one or more VRIs.

Specific content of the interventions was determined and all VRIs used a biopsychosocial approach in their intervention, but differed in the components primarily focused upon. VRI1 focused primarily on physiological improvement by means of an extensive workout scheme. Patients in the VRI1 group completed, on average, $57 \%$ of the planned physical training sessions. Even though this was stated as 'fairly' complete, only four patients completed over $75 \%$ of the training sessions planned. It appears that keeping up with a training frequency of three times a week (each $2.5 \mathrm{~h}$ ) for 18 weeks is a difficult task in this population. Furthermore, informal conversations about patient's feelings, thoughts and concerns about their illness and the training were not detailed in the VRI1 protocol, but were conducted in all patients. The content of VRI2 was individually tailored to patients' needs. The intervention focused on physiological adaptation (by physical training) and on psychosocial functioning (by cognitive behavioural sessions in groups and in individuals) and were 'fairly' or 'fully' completed. VRI3 focused on cognitive behavioural therapy on physical, personal and social/work level, provided mostly in group sessions. These components were delivered as planned. However, completeness of partner sessions was below $50 \%$. This figure may be misleading because the sessions were non-compulsory, and it is unknown whether the partner sessions were planned for each patient.

In this study, patients enrolled in one of the VRIs by 'normal' enrolment procedures. The VRIs have different ways of recruiting their clients; in VRI2 and VRI3, occupational physicians often refer sick-listed workers to the institutions. The clients of VRI1 are often advised to visit the institution by people in their social environment and/or caregivers, but are self-referred. Consequently, clients in VRI1 have to finance the treatment themselves, whereas in VRI2 and VRI3 the employer of the clients pays for the treatment. Therefore, the VRIs could have slightly different target populations, with regard to motivation and employment status. Nevertheless, the current results on patient's personal goals show that the content of the interventions individually match these individual goals.

Despite the differences in content, fatigue complaints decreased significantly at completion and at three months follow-up in all interventions. At baseline, mean CIS scores were around 100, only slightly lower than CIS scores in CFS patients $($ mean=113) [36]. After treatment and three months later, CIS scores diminished to below the cut-off point indicating chronic fatigue [36] ( $\mathrm{CIS}=76$ ), but were still higher compared with the Dutch working population $($ mean $=47)$ [36]. As for work participation, mean percentage return-to-work rose to over $80 \%$ at $\mathrm{t} 2$ in all VRIs. Remarkably, this is even the case in the VRI1 group, in which return-to-work was not a specific component of the intervention. However, work participation in the VRI1 sample was higher at baseline compared with VRI2 and VRI3 groups. After completion of the VRIs, physical functioning and mental health moved towards the scores of the Dutch population (resp. mean $=83$ and mean=77) [37]. Social functioning improved significantly only in the VRI3 group. Reasons for this single improvement may lie in involvement of patient's social environment (e.g. 
partners, family, workplace) in the intervention more than in the other VRIs. However, this is a small improvement (from 51 to 56), and scores were lower compared with the Dutch norm figures (mean=84) [37], it may be hypothesised that improvement in social functioning requires more time, especially in patients with longstanding disabling fatigue. As for heart rate variability, both patients in VRI1 and VRI2 showed significant improvements in HF power after treatment. This reflects an increase of parasympathetic activity of the autonomic nervous system. Few if any studies have investigated the effects of vocational interventions on $\mathrm{HRV}$ in fatigued patients. In healthy individuals and coronary patients, aerobic exercise, comparable to physical exercise in VRI1 and VRI2, significantly increases HF power.

The results of the current study are in line with previous research. In CFS patients, biopsychosocial interventions showed positive effects on physical, psychological and quality of life outcomes [9]. In different populations, that is, in patients with upper extremity musculoskeletal disorders [38], low back pain [39] and patients on long-term sick leave [40], multidisciplinary biopsychosocial rehabilitation showed positive effects on work participation, as well. However, few if any studies have evaluated biopsychosocial interventions in practice on work participation in fatigued patients. The VRIs in this study were not specifically designed for fatigue patients. Patients with different diseases and disabilities were treated as well.

In this study, interventions were evaluated in a real-life setting. Outpatient institutions in the Netherlands have to deal with issues such as creating contracts with clients and employers, competition with other institutions and protection of their product. Therefore, as in all business enterprises, they have financial motives in addition to providing the best care to the patient. Consequently, conducting a randomised controlled study was not feasible. However, these outpatient interventions were already practiced in real-life situations, with their own patient population and carried out by experienced trainers and supervisors. It is therefore of great importance to evaluate the outcomes of these everyday practices with many fatigued patients seeking help. Because of its uncontrolled design, the results of this study cannot directly be attributed to the interventions. However, a number of measures were taken to strengthen the design. Firstly, the VRIs content and theory was specified and outcomes were selected close to the interventions and were measured using reliable and validated outcomes variables. The results pointed in the same direction. This was shown, by longitudinal data, as well as in the process evaluation which confirmed that the VRIs were also conducted as planned. Secondly, the outcomes variables which were thought to be affected by the VRIs showed positive results. The outcomes variables which were thought to be not or less affected by the VRIs (e.g. social functioning in VRI1 and VRI2) did not show positive results. Thirdly, by using triangulation of methods, different perspectives were taken into account and we found that patient's interpretation of the outcomes did not differ from the researcher's perspective. Finally, the patients within this study were suffered from disabling fatigue for many years on average. Given the longstanding nature of fatigue complaints and perceived disabilities, spontaneous recovery of these problems was unlikely. Considering the above mentioned points, we believe that the outcomes of this study may be attributed to the interventions as provided by the outpatient institutions in a population of prolonged fatigue patients with participation problems.

The results of this study therefore suggest that these three VRIs, guided by principles of the biopsychosocial model, provide significant and clinically relevant outcomes regarding decreased fatigue symptoms and improved functioning and participation in patients with prolonged fatigue up to three months after intervention. It is therefore recommended that biopsychosocial interventions (patienttailored) be used for patients with disabling prolonged fatigue complaints. The results are of importance for occupational physicians, among others. Occupational physicians play an important role in preventing and managing sickness absence through referral to VRIs. It is, however, unclear whether the positive short-term outcomes obtained in this study will sustain over time. Research into durability of these outcomes will be of importance for those involved in treating this population and is therefore recommended.

Acknowledgments This study was financially supported by the Dutch Ministry of Social Affairs and Employment and the Dutch Ministry of Health, Welfare and Sports. The authors would like to thank the outpatient institutions for providing the patients for this study and for realisation of the interventions.

Open Access This article is distributed under the terms of the Creative Commons Attribution Noncommercial License which permits any noncommercial use, distribution, and reproduction in any medium, provided the original author(s) and source are credited.

\section{References}

1. Mohren D, Jansen N, van Amelsvoort L, Kant IJ. An Epidemiological Approach of Fatigue and Work. Experiences from the Maastricht Cohort Study. Universiteit Maastricht; 2007.

2. Bültmann U, Kant I, Kasl SV, Beurskens AJ, van den Brandt PA. Fatigue and psychological distress in the working population: psychometrics, prevalence, and correlates. J Psychosom Res. 2002;52:445-52.

3. Pawlikowska T, Chalder T, Hirsch SR, Wallace P, Wright DJ, Wessely SC. Population based study of fatigue and psychological distress. BMJ. 1994;308:763-6.

4. Wessely S. The epidemiology of chronic fatigue syndrome. Epidemiol Rev. 1995;17:139-51. 
5. Lewis G, Wessely S. The epidemiology of fatigue: more questions than answers. J Epidemiol Community Health. 1992;46:92-7.

6. Waddell G. Volvo award in clinical sciences. A new clinical model for the treatment of low-back pain. Spine (Phila Pa 1976). 1987;12:632-44.

7. Engel GL. The need for a new medical model: a challenge for biomedicine. Science. 1977;196:129-36.

8. Van Houdenhove B, Luyten P. Customizing treatment of chronic fatigue syndrome and fibromyalgia: the role of perpetuating factors. Psychosomatics. 2008;49:470-7.

9. Price JR, Mitchell E, Tidy E, Hunot V. Cognitive behaviour therapy for chronic fatigue syndrome in adults. Cochrane Database Syst Rev 2008; CD001027.

10. McEwen BS. Protective and damaging effects of stress mediators. N Engl J Med. 1998;15:171-9.

11. Papousek I, Schulter G, Premsberger E. Dissociated autonomic regulation during stress and physical complaints. J Psychosom Res. 2002;52:257-66.

12. Sluiter JK, Frings-Dresen MH, van der Beek AJ, Meijman TF. The relation between work-induced neuroendocrine reactivity and recovery, subjective need for recovery, and health status. J Psychosom Res. 2001;50:29-37.

13. Cleare AJ. The neuroendocrinology of chronic fatigue syndrome. Endocr Rev. 2003;24:236-52.

14. Huibers MJ, Bleijenberg G, van Amelsvoort LG, Beurskens AJ, van Schayck CP, Bazelmans E, et al. Predictors of outcome in fatigued employees on sick leave: results from a randomised trial. J Psychosom Res. 2004;57:443-9.

15. Vercoulen JH, Swanink CM, Galama JM, Fennis JF, Jongen PJ, Hommes OR, et al. The persistence of fatigue in chronic fatigue syndrome and multiple sclerosis: development of a model. J Psychosom Res. 1998;45:507-17.

16. Deary V, Chalder T, Sharpe M. The cognitive behavioural model of medically unexplained symptoms: a theoretical and empirical review. Clin Psychol Rev. 2007;27:781-97.

17. Van Houdenhove B, Luyten P. Treatment of chronic fatigue syndrome: how to find a 'new equilibrium'? Patient Educ Couns. 2009;77:153-4.

18. Goedendorp MM, Gielissen MF, Verhagen CA, Bleijenberg G. sychosocial interventions for reducing fatigue during cancer treatment in adults. Cochrane Database Syst Rev 2009; CD006953.

19. Goudsmit EM, Ho-Yen DO, Dancey CP. Learning to cope with chronic illness. Efficacy of a multi-component treatment for people with chronic fatigue syndrome. Patient Educ Couns. 2009;77:231-6.

20. Joosen M, Sluiter J, Joling C, Frings-Dresen M. Evaluation of the effects of a training programme for patients with prolonged fatigue on physiological parameters and fatigue complaints. Int $\mathrm{J}$ Occup Med Environ Health. 2008;21:237-46.

21. Ridsdale L, Darbishire L, Seed PT. Is graded exercise better than cognitive behaviour therapy for fatigue? A UK randomized trial in primary care. Psychol Med. 2004;34:37-49.

22. Joosen MCW, Stal W, Swijenburg RH, Sluiter JK, Frings-Dresen MHW. Evaluatie van een multidisciplinair vitaliteitsprogramma bij aanhoudende vermoeidheidsklachten. (Evaluation of a multidisciplinairy treatment programme on prolonged fatigue). Tijdschr Bedr-Verzekgeneeskd. 2007;15:437-43.

23. Dooris M. Healthy settings: challenges to generating evidence of effectiveness. Health Promot Int. 2006;21:55-65.

24. Baranowski T, Stables G. Process evaluations of the 5-a-day projects. Health Educ Behav. 2000;27:157-66.
25. Vercoulen JH, Swanink CM, Fennis JF, Galama JM, van der Meer JW, Bleijenberg G. Dimensional assessment of chronic fatigue syndrome. J Psychosom Res. 1994;38:383-92.

26. Beurskens AJ, Bültmann U, Kant I, Vercoulen JH, Bleijenberg G, Swaen GM. Fatigue among working people: validity of a questionnaire measure. Occup Environ Med. 2000;57:353-7.

27. van der Zee KI, Sanderman R. Het meten van de algemene gezondheidstoestand met de RAND-36. Een handleiding. (Measuring general health status with the RAND-36. Users Manual). Rijksuniversiteit Groningen: Noordelijk Centrum voor Gezondheidsvraagstukken; 1993.

28. Ware JE, Sherbourne CD. 36-item short-form health survey (SF36). Conceptual framework and item selection. Med Care. 1992;30:473-83.

29. van Veldhoven M, Broersen S. Measurement quality and validity of the "need for recovery scale". Occup Environ Med. 2003;60 Suppl 1:i3-9.

30. de Croon EM, Sluiter JK, Frings-Dresen MH. Psychometric properties of the need for recovery after work scale: test-retest reliability and sensitivity to detect change. Occup Environ Med. 2006;63:202-6.

31. Tuomi K, Ilmarinen J, Jahkola A, Katajarinne L, Tulkki A. Work Ability Index. Helsinki: Finnish Institute of Occupational Health; 1997.

32. Langelaan S, Bakker AB, Schaufeli WB, Van RW, van Doornen LJ. Is burnout related to allostatic load? Int J Behav Med. 2007;14:213-21.

33. Berntson GG, Bigger Jr JT, Eckberg DL, Grossman P, Kaufmann PG, Malik M, et al. Heart rate variability: origins, methods, and interpretive caveats. Psychophysiology. 1997;34:623-48.

34. The Task Force of the European Society of Cardiology, The North American Society of Pacing and Electrophysiology. Heart rate variability. Standards of measurement, physiological interpretation, and clinical use. Eur Heart J. 1996;17:354-81.

35. Sluiter JK, Guijt AM, Frings-Dresen MH. Reproducibility and validity of heart rate variability and respiration rate measurements in participants with prolonged fatigue complaints. Int Arch Occup Environ Health. 2009;66:305-11.

36. Bültmann U, de Vries M, Beurskens AJ, Bleijenberg G, Vercoulen JH, Kant I. Measurement of prolonged fatigue in the working population: determination of a cutoff point for the checklist individual strength. J Occup Health Psychol. 2000;5:411-6.

37. Aaronson NK, Muller M, Cohen PD, Essink-Bot ML, Fekkes M, Sanderman R, et al. Translation, validation, and norming of the Dutch language version of the SF-36 Health Survey in community and chronic disease populations. J Clin Epidemiol. 1998;51:1055-68.

38. Karjalainen K, Malmivaara A, van TM, Roine R, Jauhiainen M, Hurri $\mathrm{H}$, et al. Multidisciplinary biopsychosocial rehabilitation for neck and shoulder pain among working age adults. Cochrane Database Syst Rev 2003; CD002194.

39. Karjalainen K, Malmivaara A, van TM, Roine R, Jauhiainen M, Hurri $\mathrm{H}$, et al. Multidisciplinary biopsychosocial rehabilitation for subacute low back pain among working age adults. Cochrane Database Syst Rev 2003; CD002193.

40. Braathen TN, Veiersted KB, Heggenes J. Improved work ability and return to work following vocational multidisciplinary rehabilitation of subjects on long-term sick leave. J Rehabil Med. 2007;39:493-9. 